\title{
Multi-sublinear operators generated by multilinear Calderón-Zygmund operators on product generalized Morrey spaces
}

\author{
Afaq F. Ismayilova ${ }^{\star}$ - Fatayi A. Isayev
}

Received: 08.08.2019 / Revised: 08.03.2020 / Accepted: 05.06.2020

\begin{abstract}
In this paper, we established the boundedness for a large class of multi-sublinear operators $T_{m}$ generated by multilinear Calderón-Zygmund operators on product generalized Morrey spaces $\mathcal{M}_{p_{1}, \varphi_{1}}\left(\mathbb{R}^{n}\right) \times \ldots \times \mathcal{M}_{p_{m}, \varphi_{m}}\left(\mathbb{R}^{n}\right)$. We find the sufficient conditions on $\left(\varphi_{1}, \ldots, \varphi_{m}, \varphi\right)$ which ensures the boundedness of the operators $T_{m}$ from $\mathcal{M}_{p_{1}, \varphi_{1}}\left(\mathbb{R}^{n}\right) \times \ldots \times \mathcal{M}_{p_{m}, \varphi_{m}}\left(\mathbb{R}^{n}\right)$ to $\mathcal{M}_{p, \varphi}\left(\mathbb{R}^{n}\right)$ for $1 / p=1 / p_{1}+\ldots+1 / p_{m}$. The multi-sublinear operators under consideration contain integral operators of harmonic analysis such as multi-sublinear maximal operator $\mathcal{M}_{m}$, multilinear Calderón-Zygmund operators $\mathcal{T}_{m}$, etc.
\end{abstract}

Keywords. Multi-sublinear maximal operator; multilinear Calderón-Zygmund operator; product generalized Morrey space.

Mathematics Subject Classification (2010): 42B20, 42B25, 42B35.

\section{Introduction}

Multilinear Calderón-Zygmund theory is a natural generalization of the linear case. The initial work on the class of multilinear Calderón-Zygmund operators was done by Coifman and Meyer in [2] and was later systematically studied by Grafakos and Torres in [9,10].

The classical Morrey spaces, introduced by Morrey [23] in 1938, have been studied intensively by various authors and together with Lebesgue spaces play an important role in the theory of partial differential equations. Although such spaces allow to describe local properties of functions better than Lebesgue spaces, they have some unpleasant issues. It is well known that Morrey spaces are non separable and that the usual classes of nice functions are not dense in such spaces. Moreover, various Morrey spaces are defined in the process of study. Guliyev, Mizuhara and Nakai [11,21,24] introduced generalized Morrey spaces $\mathcal{M}_{p, \varphi}\left(\mathbb{R}^{n}\right)$ (see, also $\left.[12,13,17,26]\right)$. In [13] is defined the generalized Morrey spaces $\mathcal{M}_{p, \varphi}$ with normalized norm

$$
\|f\|_{\mathcal{M}_{p, \varphi}} \equiv \sup _{x \in \mathbb{R}^{n}, r>0} \varphi(x, r)^{-1}|B(x, r)|^{-1 / p}\|f\|_{L_{p}(B(x, r))},
$$

\footnotetext{
* Corresponding author
}

A.F. Ismayilova

Azerbaijan University of Cooperation, Baku, Azerbaijan

E-mail: afaismayilova28@gmail.com

F.A. Isayev

Institute of Mathematics and Mechanics of NAS of Azerbaijan, Baku, Azerbaijan

E-mail: isayevfatai@yahoo.com 
where the function $\varphi$ is a positive measurable function on $\mathbb{R}^{n} \times(0, \infty)$. Here and everywhere in the sequel $B(x, r)$ is the ball in $\mathbb{R}^{n}$ of radius $r$ centered at $x$ and $|B(x, r)|=v_{n} r^{n}$ is its Lebesgue measure, where $v_{n}$ is the volume of the unit ball in $\mathbb{R}^{n}$. In [11] Guliyev also studied the boundedness of the classical operators in these spaces $\mathcal{M}_{p, \varphi}$, see also $[3-8,18$, $19,25]$.

For $x \in \mathbb{R}^{n}$ and $r>0$, we denote by $B(x, r)$ the open ball centered at $x$ of radius $r$, and by ${ }^{\mathrm{C}} B(x, r)$ denote its complement. Let $|B(x, r)|$ be the Lebesgue measure of the ball $B(x, r)$. We denote by $\vec{f}$ the $m$-tuple $\left(f_{1}, f_{2}, \ldots, f_{m}\right), \vec{y}=\left(y_{1}, \ldots, y_{n}\right)$ and $d \vec{y}=$ $d y_{1} \cdots d y_{n}$.

Let $\vec{f} \in L_{p_{1}}^{\text {loc }}\left(\mathbb{R}^{n}\right) \times \ldots \times L_{p_{m}}^{\text {loc }}\left(\mathbb{R}^{n}\right)$. The multi-sublinear maximal operator $M_{m}$ is defined by

$$
M_{m}(\vec{f})(x)=\sup _{r>0} \prod_{i=1}^{m} \frac{1}{|B(x, r)|} \int_{B(x, r)}\left|f_{i}\left(y_{i}\right)\right| d y_{i} .
$$

In [10] Grafakos and Torres studied the multilinear Calderón-Zygmund operator which can be written for $x \notin \cap_{j=1}^{m} \operatorname{supp} f_{j}$ as

$$
K_{m}(\vec{f})(x)=\int_{\left(\mathbb{R}^{n}\right)^{m}} K\left(x, y_{1}, \ldots, y_{m}\right) f_{1}\left(y_{1}\right) \ldots f_{m}\left(y_{m}\right) d y_{1} d y_{2} \ldots d y_{m},
$$

where $K\left(x, y_{1}, \ldots, y_{m}\right)$ is the kernel function defined of the diagonal $x=y_{1}=\ldots y_{m}=$ in $\left(\mathbb{R}^{n}\right)^{m+1}$ satisfying

$$
\left|K\left(y_{0}, y_{1}, \ldots, y_{m}\right)\right| \leq c_{1}\left(\sum_{k, l=0}^{m}\left|y_{k}-y_{l}\right|\right)^{-m n},
$$

and whenever $2\left|y_{j}-y_{j}^{\prime}\right| \leq \frac{1}{2} \max _{0 \leq k \leq m}\left|y_{j}-y_{k}\right|$,

$$
\left|K\left(y_{0}, \ldots, y_{j}, \ldots, y_{m}\right)-K\left(y_{0}, \ldots, y_{j}^{\prime}, \ldots, y_{m}\right)\right| \leq \frac{c_{1}\left|y_{j}-y_{j}^{\prime}\right|^{\epsilon}}{\left(\sum_{k, l=0}^{m}\left|y_{k}-y_{l}\right|\right)^{m n+\epsilon}}
$$

for some $\epsilon>0$ and all $0 \leq j \leq m$. Grafakos and Torres [10] proved that the operator $K_{m}(\vec{f})$ is bounded from $L_{p_{1}}\left(\mathbb{R}^{n}\right) \times \ldots \times L_{p_{m}}\left(\mathbb{R}^{n}\right)$ to $L_{p}\left(\mathbb{R}^{n}\right)$ for $p_{i}>1(i=1, \ldots, m)$ and $1 / p=1 / p_{1}+\ldots+1 / p_{m}$, and bounded from $L_{1}\left(\mathbb{R}^{n}\right) \times \ldots \times L_{1}\left(\mathbb{R}^{n}\right)$ to $L_{\frac{1}{m}, \infty}\left(\mathbb{R}^{n}\right)$.

It is well known that multi-sublinear maximal operator and multilinear Calderón-Zygmund operators play an important role in harmonic analysis (see [1,10,22]).

Suppose that $T_{m}$ represents a multilinear or a multi-sublinear operator, which satisfies that for any $\vec{f} \in L_{1}\left(\mathbb{R}^{n}\right) \times \ldots \times L_{1}\left(\mathbb{R}^{n}\right)$ with compact support and $x \notin \cap_{j=1}^{m} \operatorname{supp} f_{j}$

$$
\left|T_{m}(\vec{f})(x)\right| \leq c_{0} \int_{\left(\mathbb{R}^{n}\right)^{m}} \frac{\left|f_{1}\left(y_{1}\right) \ldots f_{m}\left(y_{m}\right)\right|}{\left|\left(x-y_{1}, \ldots, x-y_{m}\right)\right|^{m n}} d y_{1} d y_{2} \ldots d y_{m}
$$

where $c_{0}$ is independent of $\vec{f}$ and $x$.

The condition (1.1) is satisfied by many interesting operators in harmonic analysis, such as the multilinear Calderón-Zygmund operators, multi-sublinear maximal operator, and so on (see $[10,20]$ for details). 
In this work, we prove the boundedness of the multi-sublinear operator $T_{m}$ satisfies the condition (1.1) generated by multilinear Calderón-Zygmund operator from product generalized Morrey space $\mathcal{M}_{p_{1}, \varphi_{1}} \times \ldots \times \mathcal{M}_{p_{m}, \varphi_{m}}$ to $\mathcal{M}_{p, \varphi}$, if $1<p_{1}, \ldots, p_{m}<\infty$ and $1 / p=1 / p_{1}+\cdots+1 / p_{m}$, and from the space $\mathcal{M}_{p_{1}, \varphi_{1}} \times \ldots \times \mathcal{M}_{p_{m}, \varphi_{m}}$ to the weak space $W \mathcal{M}_{1, \varphi}$, if $1 \leq p_{1}, \ldots, p_{m}<\infty, 1 / p=1 / p_{1}+\cdots+1 / p_{m}$ and at least one $p_{i}$ equals one (Theorem 2.3). Finally, as applications we apply this result to several particular operators such as the multi-sublinear maximal operator and multilinear Calderón-Zygmund operator.

By $A \lesssim B$ we mean that $A \leq C B$ with some positive constant $C$ independent of appropriate quantities. If $A \lesssim B$ and $B \lesssim A$, we write $A \approx B$ and say that $A$ and $B$ are equivalent.

\section{Main Results}

In this section, we will discuss the boundedness properties of multi-sublinear operators $T_{m}$ generated by multilinear Calderón-Zygmund operators on product generalized Morrey spaces $\mathcal{M}_{p_{1}, \varphi_{1}}\left(\mathbb{R}^{n}\right) \times \ldots \times \mathcal{M}_{p_{m}, \varphi_{m}}\left(\mathbb{R}^{n}\right)$.

We find it convenient to define the generalized Morrey spaces in the form as follows.

Definition 2.1 Let $\varphi(x, r)$ be a positive measurable function on $\mathbb{R}^{n} \times(0, \infty)$ and $1 \leq$ $p<\infty$. We denote by $M_{p, \varphi} \equiv M_{p, \varphi}\left(\mathbb{R}^{n}\right)$ the generalized Morrey space, the space of all functions $f \in L_{p}^{\text {loc }}\left(\mathbb{R}^{n}\right)$ with finite quasinorm

$$
\|f\|_{M_{p, \varphi}}=\sup _{x \in \mathbb{R}^{n}, r>0} \varphi(x, r)^{-1}|B(x, r)|^{-\frac{1}{p}}\|f\|_{L_{p}(B(x, r))} .
$$

Also by $W M_{p, \varphi} \equiv W M_{p, \varphi}\left(\mathbb{R}^{n}\right)$ we denote the weak generalized Morrey space of all functions $f \in W L_{p}^{\text {loc }}\left(\mathbb{R}^{n}\right)$ for which

$$
\|f\|_{W M_{p, \varphi}}=\sup _{x \in \mathbb{R}^{n}, r>0} \varphi(x, r)^{-1}|B(x, r)|^{-\frac{1}{p}}\|f\|_{W L_{p}(B(x, r))}<\infty .
$$

Lemma 2.1 [4] Let $\varphi(x, r)$ be a positive measurable function on $\mathbb{R}^{n} \times(0, \infty)$.

(i) If

$$
\sup _{t<r<\infty} \frac{r^{-\frac{n}{p}}}{\varphi(x, r)}=\infty \quad \text { for some } t>0 \text { and for all } x \in \mathbb{R}^{n},
$$

(ii) If

then $M_{p, \varphi}\left(\mathbb{R}^{n}\right)=\Theta$.

$$
\sup _{0<r<\tau} \varphi(x, r)^{-1}=\infty \quad \text { for some } \tau>0 \text { and for all } x \in \mathbb{R}^{n},
$$

then $M_{p, \varphi}\left(\mathbb{R}^{n}\right)=\Theta$.

Remark 2.1 We denote by $\Omega_{p}$ the sets of all positive measurable functions $\varphi$ on $\mathbb{R}^{n} \times$ $(0, \infty)$ such that for all $t>0$,

$$
\sup _{x \in \mathbb{R}^{n}}\left\|\frac{r^{-\frac{n}{p}}}{\varphi(x, r)}\right\|_{L_{\infty}(t, \infty)}<\infty, \quad \text { and } \sup _{x \in \mathbb{R}^{n}}\left\|\varphi(x, r)^{-1}\right\|_{L_{\infty}(0, t)}<\infty,
$$

respectively. In what follows, keeping in mind Lemma 2.1, we always assume that $\varphi \in \Omega_{p}$. 
We will use the following statements on the boundedness of the weighted Hardy operator

$$
H_{w} g(r):=\int_{r}^{\infty} g(t) w(t) d t, 0<t<\infty,
$$

where $w$ is a fixed function non-negative and measurable on $(0, \infty)$.

The following theorem was proved in [14] (see also [16]).

Theorem 2.1 [14] Let $v_{1}, v_{2}$ and $w$ be positive almost everywhere and measurable functions on $(0, \infty)$. The inequality

$$
\underset{r>0}{\operatorname{ess} \sup _{2}} v_{2}(r) H_{w} g(r) \leq C \underset{r>0}{\operatorname{ess} \sup } v_{1}(r) g(r)
$$

holds for some $C>0$ for all non-negative and non-decreasing $g$ on $(0, \infty)$ if and only if

$$
B:=\sup _{r>0} v_{2}(r) \int_{r}^{\infty} \frac{w(t) d t}{\sup _{t<s<\infty} v_{1}(s)}<\infty .
$$

Moreover, the value $C=B$ is the best constant for (2.3).

Remark 2.2 In (2.3) - (2.4) it is assumed that $0 \cdot \infty=0$.

In the following lemma we get Guliyev local estimate (see, for example, [11-13] in the case $m=1$ and [15] in the case $m>1$ ) for the operator $T_{m}$.

Theorem 2.2 Let $1 \leq p_{1}, \ldots, p_{m}<\infty$ and $1 / p=1 / p_{1}+\cdots+1 / p_{m}$. Let $T_{m}$ be a multi-sublinear operator which satisfies the condition (1.1) bounded from $L_{p_{1}}\left(\mathbb{R}^{n}\right) \times \ldots \times$ $L_{p_{m}}\left(\mathbb{R}^{n}\right)$ to $L_{p}\left(\mathbb{R}^{n}\right)$ for $p_{i}>1, i=1, \ldots, m$, and bounded from $L_{p_{1}}\left(\mathbb{R}^{n}\right) \times \ldots \times L_{p_{m}}\left(\mathbb{R}^{n}\right)$ to $W L_{p}\left(\mathbb{R}^{n}\right)$ for $p_{i} \geq 1, i=1, \ldots, m$.

Then, for $1<p_{1}, \ldots, p_{m}<\infty$ the inequality

$$
\left\|T_{m}(\vec{f})\right\|_{L_{p}\left(B\left(x_{0}, r\right)\right)} \lesssim r^{\frac{n}{p}} \prod_{i=1}^{m} \int_{2 r}^{\infty} t^{-\frac{n}{p_{i}}-1}\left\|f_{i}\right\|_{L_{p_{i}}\left(B\left(x_{0}, t\right)\right)} d t
$$

holds for any ball $B\left(x_{0}, r\right)$ and for all $\vec{f} \in L_{p_{1}}^{\text {loc }}\left(\mathbb{R}^{n}\right) \times \ldots \times L_{p_{m}}^{\text {loc }}\left(\mathbb{R}^{n}\right)$.

Moreover, if at least one $p_{i}$ equals one, the inequality

$$
\left\|T_{m}(\vec{f})\right\|_{W L_{p}\left(B\left(x_{0}, r\right)\right)} \lesssim r^{\frac{n}{p}} \prod_{i=1}^{m} \int_{2 r}^{\infty} t^{-\frac{n}{p_{i}}-1}\left\|f_{i}\right\|_{L_{p_{i}}\left(B\left(x_{0}, t\right)\right)} d t
$$

holds for any ball $B\left(x_{0}, r\right)$ and for all $\vec{f} \in L_{p_{1}}^{\text {loc }}\left(\mathbb{R}^{n}\right) \times \ldots \times L_{p_{m}}^{\text {loc }}\left(\mathbb{R}^{n}\right)$.

Proof. Let $1<p_{1}, \ldots, p_{m}<\infty$ and $1 / p=1 / p_{1}+\cdots+1 / p_{m}$. For arbitrary $x_{0} \in \mathbb{R}^{n}$, set $B=B\left(x_{0}, r\right)$ for the ball centered at $x_{0}$ and of radius $r, 2 B=B\left(x_{0}, 2 r\right)$. We represent $\vec{f}=\left(f_{1}, \ldots, f_{m}\right)$ as

$$
f_{j}=f_{j}^{0}+f_{j}^{\infty}, \quad f_{j}^{0}=f_{j} \chi_{2 B}, \quad f_{j}^{\infty}=f_{j} \chi_{\mathrm{c}_{(2 B)}}, \quad j=1, \ldots, m .
$$

Then we split $T_{m}(\vec{f})$ as follows

$$
\left|T_{m}(\vec{f})(x)\right| \leq c_{0}\left|T_{m}\left(f_{1}^{0}, \ldots, f_{m}^{0}\right)(x)\right|+\left|\sum_{\beta_{1}, \ldots, \beta_{m}}^{\prime} T_{m}\left(f_{1}^{\beta_{1}}, \ldots, f_{m}^{\beta_{m}}\right)(x)\right|,
$$


where $\beta_{1}, \ldots, \beta_{m} \in\{0, \infty\}$ and each term of $\sum^{\prime}$ contains at least $\beta_{i} \neq 0$. Then,

$$
\begin{aligned}
\left\|T_{m}(\vec{f})\right\|_{L_{p}(B(x, r))} & \leq\left\|T_{m}\left(f_{1}^{0}, \ldots, f_{m}^{0}\right)\right\|_{L_{p}(B(x, r))} \\
& +\left\|\sum_{\beta_{1}, \ldots, \beta_{m}}^{\prime} T_{m}\left(f_{1}^{\beta_{1}}, \ldots, f_{m}^{\beta_{m}}\right)\right\|_{L_{p}(B(x, r))} \leq I+I I .
\end{aligned}
$$

For $I$, by the boundedness of $T_{m}$ from product $L_{p_{1}}\left(\mathbb{R}^{n}\right) \times \ldots \times L_{p_{m}}\left(\mathbb{R}^{n}\right)$ to $L_{p}\left(\mathbb{R}^{n}\right)$ with $1 / p=1 / p_{1}+\ldots+1 / p_{m}$ for each $p_{i}>1(i=1, \ldots, m)$, we have,

$$
\begin{aligned}
\left\|T_{m}\left(\overrightarrow{f^{0}}\right)\right\|_{L_{p}(B(x, r))} & \leq\left\|T_{m}\left(\overrightarrow{f^{0}}\right)\right\|_{L_{p}\left(\mathbb{R}^{n}\right)} \\
& \lesssim \prod_{i=1}^{m}\left\|f_{i}^{0}\right\|_{L_{p_{i}}\left(\mathbb{R}^{n}\right)} \lesssim \prod_{i=1}^{m}\left\|f_{i}\right\|_{L_{p_{i}}(B(x, 2 r))} .
\end{aligned}
$$

Taking into account that

$$
\left\|f_{i}\right\|_{L_{p_{i}}(B(x, 2 r))} \lesssim r^{\frac{n}{p_{i}}} \int_{2 r}^{\infty} t^{-\frac{n}{p_{i}}-1}\left\|f_{i}\right\|_{L_{p_{i}}(B(x, t))} d t, \quad i=1, \ldots, m
$$

we get

$$
\left\|T_{m}\left(\overrightarrow{f^{0}}\right)\right\|_{L_{p}(B(x, r))} \lesssim r^{\frac{n}{p}} \prod_{i=1}^{m} \int_{2 r}^{\infty} t^{-\frac{n}{p_{i}}-1}\left\|f_{i}\right\|_{L_{p_{i}}(B(x, t))} d t .
$$

For $I I$, first we consider the case $\beta_{1}=\cdots=\beta_{m}=\infty$.

When $\left|x-y_{i}\right| \leq r,\left|z-y_{i}\right| \geq 2 r$, we have $\frac{1}{2}\left|z-y_{i}\right| \leq\left|x-y_{i}\right| \leq \frac{3}{2}\left|z-y_{i}\right|$, and so by the condition (1.1) we have

$$
\begin{aligned}
\left|T_{m}\left(\overrightarrow{f^{\infty}}\right)(z)\right| & \lesssim \int_{\left({ }_{B(x, 2 r)}\right)^{m}} \frac{\left|f_{1}\left(y_{1}\right) \cdots f_{m}\left(y_{m}\right)\right|}{\left|\left(x-y_{1}, \ldots, x-y_{m}\right)\right|^{m n}} d \vec{y} \\
& \lesssim \prod_{i=1}^{m} \int_{\mathrm{c}_{B(x, 2 r)}} \frac{\left|f_{i}\left(y_{i}\right)\right|}{\left|x-y_{i}\right|^{n}} d y_{i}
\end{aligned}
$$

and

$$
\begin{aligned}
\left\|T_{m}\left(\overrightarrow{f^{\infty}}\right)\right\|_{L_{p}(B(x, r))} & \leq \prod_{i=1}^{m} \int_{\mathrm{c}_{B(x, 2 r)}} \frac{\left|f_{i}\left(y_{i}\right)\right|}{\left|x-y_{i}\right|^{n}} d y_{i}\left\|\chi_{B(x, r)}\right\|_{L_{p}\left(\mathbb{R}^{n}\right)} \\
& \lesssim r^{\frac{n}{p}} \prod_{i=1}^{m} \int_{\mathrm{c}_{B(x, 2 r)}} \frac{\left|f_{i}\left(y_{i}\right)\right|}{\left|x-y_{i}\right|^{n}} d y_{i} .
\end{aligned}
$$

By Fubini's theorem we have

$$
\begin{aligned}
\int_{\mathrm{c}_{B(x, 2 r)}} \frac{\left|f_{i}\left(y_{i}\right)\right|}{\left|x-y_{i}\right|^{n}} d y_{i} & \approx \int_{\mathrm{c}_{B(x, 2 r)}}\left|f_{i}\left(y_{i}\right)\right| \int_{\left|x_{0}-y_{i}\right|}^{\infty} \frac{d t}{t^{n+1}} d y_{i} \\
& \approx \int_{2 r}^{\infty} \int_{2 r \leq\left|x_{0}-y_{i}\right|<t}\left|f_{i}\left(y_{i}\right)\right| d y_{i} \frac{d t}{t^{n+1}} \\
& \lesssim \int_{2 r}^{\infty} \int_{B\left(x_{0}, t\right)}\left|f_{i}\left(y_{i}\right)\right| d y_{i} \frac{d t}{t^{n+1}} .
\end{aligned}
$$


Applying Hölder's inequality, we get

$$
\int_{\mathrm{c}_{B(x, 2 r)}} \frac{\left|f_{i}\left(y_{i}\right)\right|}{\left|x-y_{i}\right|^{n}} d y_{i} \lesssim \int_{2 r}^{\infty} t^{-\frac{n}{p_{i}}-1}\left\|f_{i}\right\|_{L_{p_{i}}(B(x, t))} d t .
$$

Moreover, for all $p_{i} \in[1, \infty), i=1, \ldots, m$ the inequality

$$
\left\|T_{m}\left(\overrightarrow{f^{\infty}}\right)\right\|_{L_{p}(B(x, r))} \lesssim r^{\frac{n}{p}} \prod_{i=1}^{m} \int_{2 r}^{\infty} t^{-\frac{n}{p_{i}}-1}\left\|f_{i}\right\|_{L_{p_{i}}(B(x, t))} d t
$$

is valid.

Next we consider the case that some $\alpha_{i}=0$ and other $\alpha_{j}=\infty$. To this end we may assume that $\alpha_{1}=\alpha_{2}=\infty$ and $\alpha_{3}=\cdots=\alpha_{m}=0$. Recall the condition (1.1) and the fact that $\left|x-y_{i}\right| \approx\left|z-y_{i}\right|$ for $z \in B(x, r)$ and $y_{i} \in{ }^{\complement} B(x, 2 r)$, we have that

$$
\begin{aligned}
& T\left(f_{1}^{\infty}, f_{2}^{\infty}, f_{3}^{0}, \ldots, f_{m}^{0}\right)(z) \\
& \lesssim \int_{{ }^{\mathfrak{C}_{B(x, 2 r)} \times{ }^{\mathrm{C}} B(x, 2 r)}} \frac{\left|f_{1}\left(y_{1}\right)\right|\left|f_{2}\left(y_{2}\right)\right|}{\left(\left|x-y_{1}\right|+\left|x-y_{2}\right|\right)^{m n}} d y_{1} d y_{2} \prod_{i=3}^{m} \int_{B(x, 2 r)}\left|f_{i}\left(y_{i}\right)\right| d y_{i} \\
& \lesssim \int_{\mathfrak{c}_{B(x, 2 r)}} \frac{\left|f_{1}\left(y_{1}\right)\right|}{\left|x-y_{1}\right|^{n}} d y_{1} \int_{\mathfrak{c}_{B(x, 2 r)}} \frac{\left|f_{2}\left(y_{2}\right)\right|}{\left|x-y_{2}\right|^{n}} d y_{2} \prod_{i=3}^{m} \int_{B(x, 2 r)}\left|f_{i}\left(y_{i}\right)\right| d y_{i} .
\end{aligned}
$$

By the inequality (2.9) and use the Hölder's inequality for integrals, we get

$$
\begin{aligned}
& \left\|T\left(f_{1}^{\infty}, f_{2}^{\infty}, f_{3}^{0}, \ldots, f_{m}^{0}\right)\right\|_{L_{p}(B(x, r))} \\
& \lesssim r^{\frac{n}{p}} \int_{{ }^{\complement}(x, 2 r)} \frac{\left|f_{1}\left(y_{1}\right)\right|}{\left|x-y_{1}\right|^{n}} d y_{1} \int_{\complement_{B(x, 2 r)}} \frac{\left|f_{2}\left(y_{2}\right)\right|}{\left|x-y_{2}\right|^{n}} d y_{2} \prod_{i=3}^{m} \int_{B(x, 2 r)}\left|f_{i}\left(y_{i}\right)\right| d y_{i} \\
& \leq r^{\frac{n}{p}} \prod_{i=3}^{m} \int_{r}^{\infty} t^{-\frac{n}{p_{i}}-1}\left\|f_{i}\right\|_{L_{p_{i}}(B(x, t))} d t .
\end{aligned}
$$

For the proof of the inequality (2.6), by a similar argument as in the proof of (2.5) and pay attention to the fact that $\vec{f} \rightarrow T_{m}(\vec{f})$ is bounded from $L_{p_{1}}\left(\mathbb{R}^{n}\right) \times \cdots \times L_{p_{m}}\left(\mathbb{R}^{n}\right)$ to $W L_{p}\left(\mathbb{R}^{n}\right)$, we can similarly prove (2.6) and we omit the details here.

Now we give the boundedness of multi-sublinear operators generated by multilinear CalderónZygmund operators on product generalized Morrey space.

Theorem 2.3 Let $1 \leq p_{1}, \ldots, p_{m}<\infty$ with $1 / p=1 / p_{1}+\ldots+1 / p_{m}$ and $\left(\varphi_{1}, \ldots, \varphi_{m}, \varphi\right) \in$ $\Omega_{p_{1}} \times \ldots \times \Omega_{p_{1}} \times \Omega_{p}$ satisfies the condition

$$
\prod_{i=1}^{m} \int_{r}^{\infty} \frac{\underset{t<s<\infty}{\operatorname{essinf}} \varphi_{i}(x, s) s^{\frac{n}{p_{i}}}}{t^{\frac{n}{p_{i}}+1}} d t \lesssim \varphi(x, r) .
$$

Let also $T_{m}$ be a multi-sublinear operator which satisfies the condition (1.1) and bounded from $L_{p_{1}}\left(\mathbb{R}^{n}\right) \times \ldots \times L_{p_{m}}\left(\mathbb{R}^{n}\right)$ to $L_{p}\left(\mathbb{R}^{n}\right)$ for $p_{i}>1, i=1, \ldots, m$, and bounded from $L_{p_{1}}\left(\mathbb{R}^{n}\right) \times \ldots \times L_{p_{m}}\left(\mathbb{R}^{n}\right)$ to $W L_{p}\left(\mathbb{R}^{n}\right)$ for $p_{i} \geq 1, i=1, \ldots, m$. Then the operator $T_{m}$ is bounded from product space $\mathcal{M}_{p_{1}, \varphi_{1}}\left(\mathbb{R}^{n}\right) \times \ldots \times \mathcal{M}_{p_{m}, \varphi_{m}}\left(\mathbb{R}^{n}\right)$ to $\mathcal{M}_{p, \varphi}\left(\mathbb{R}^{n}\right)$ for $p_{i}>1$, $i=1, \ldots, m$, and from product space $\mathcal{M}_{p_{1}, \varphi_{1}}\left(\mathbb{R}^{n}\right) \times \ldots \times \mathcal{M}_{p_{m}, \varphi_{m}}\left(\mathbb{R}^{n}\right)$ to $W \mathcal{M}_{p, \varphi}\left(\mathbb{R}^{n}\right)$ for at least one $p_{i}$ equals one. 
Proof. Let $1<p_{1}, \ldots, p_{m}<\infty$ and $\vec{f} \in \mathcal{M}_{p_{1}, \varphi_{1}}\left(\mathbb{R}^{n}\right) \times \ldots \times \mathcal{M}_{p_{m}, \varphi_{m}}\left(\mathbb{R}^{n}\right)$. By Theorems 2.1 and 2.2 we have

$$
\begin{aligned}
\left\|T_{\alpha, m}(\vec{f})\right\|_{\mathcal{M}_{p, \varphi}} & \lesssim \sup _{x \in \mathbb{R}^{n}, r>0} \varphi(x, r)^{-1} \prod_{i=1}^{m} \int_{r}^{\infty} t^{-\frac{n}{p_{i}}-1}\left\|f_{i}\right\|_{L_{p_{i}}(B(x, t))} d t \\
& \lesssim \prod_{i=1}^{m} \sup _{x \in \mathbb{R}^{n}, r>0} \varphi_{i}(x, r)^{-1} r\left\|f_{i}\right\|_{L_{p_{i}}(B(x, r))}=\prod_{i=1}^{m}\left\|f_{i}\right\|_{M_{p_{i}, \varphi_{i}}} .
\end{aligned}
$$

When $p_{i}=1, i=1, \ldots, m$, the proof is similar and we omit the details here.

Corollary 2.1 [15] Let $1 \leq p_{1}, \ldots, p_{m}<\infty$ with $1 / p=1 / p_{1}+\ldots+1 / p_{m}$. Let also $\left(\varphi_{1}, \ldots, \varphi_{m}, \varphi\right) \in \Omega_{p_{1}} \times \ldots \times \Omega_{p_{1}} \times \Omega_{p}$ satisfies the condition (2.11). Then the operators $M_{m}$ and $K_{m}$ are bounded from product space $\mathcal{M}_{p_{1}, \varphi_{1}}\left(\mathbb{R}^{n}\right) \times \ldots \times \mathcal{M}_{p_{m}, \varphi_{m}}\left(\mathbb{R}^{n}\right)$ to $\mathcal{M}_{p, \varphi}\left(\mathbb{R}^{n}\right)$ for $p_{i}>1, i=1, \ldots, m$ and from product space $\mathcal{M}_{p_{1}, \varphi_{1}}\left(\mathbb{R}^{n}\right) \times \ldots \times$ $\mathcal{M}_{p_{m}, \varphi_{m}}\left(\mathbb{R}^{n}\right)$ to $W \mathcal{M}_{p, \varphi}\left(\mathbb{R}^{n}\right)$ for at least one $p_{i}$ equals one.

\section{Acknowledgements}

The authors thank the referee(s) for careful reading the paper and useful comments. The research of F. Isayev was partially supported by the Grant of 1st Azerbaijan-Russia Joint Grant Competition (Agreement Number No. EIF-BGM-4-RFTF1/ 2017-21/01/1-M-08).

\section{References}

1. Coifman R., Grafakos L.: Hardy spaces estimates for multilinear operators I, Rev. Math. Iberoamericana, 8, 45-68 (1992).

2. Coifman R.R., Meyer Y.: On commutators of singular integrals and bilinear singular integrals, Trans. Amer. Math. Soc. 212, 315-331 (1975).

3. Eroglu A., Hasanov A.A., Omarova M.N.: $(p, q)$-admissible multilinear fractional integral operators and their commutators in product generalized local Morrey spaces, Trans. Natl. Acad. Sci. Azerb. Ser. Phys.-Tech. Math. Sci. 36 (4), Mathematics, 77-98 (2016).

4. Eroglu, A., Guliyev, V.S., Azizov, C.V.: Characterizations for the fractional integral operators in generalized Morrey spaces on Carnot groups, Math. Notes. 102 (5-6), 722-734 (2017).

5. Eroglu A, Azizov C.V.: A note on the fractional integral operators in generalized Morrey spaces on the Heisenberg group, Trans. Natl. Acad. Sci. Azerb. Ser. Phys.-Tech. Math. Sci. 37 (1), Mathematics, 86-91 (2017).

6. Eroglu A, Isayev F.A., Namazov F.M.: Fractional integral associated to Schrdinger operator on the Heisenberg groups in vanishing generalized Morrey spaces, Trans. Natl. Acad. Sci. Azerb. Ser. Phys.-Tech. Math. Sci. 38 (4), Mathematics, 54-62 (2018).

7. Eroglu A, Azizov C.V., Guliyev, V.S.: Fractional maximal operator and its commutators in generalized Morrey spaces on Heisenberg group, Proc. Inst. Math. Mech. Natl. Acad. Sci. Azerb. 44 (2), 304-317 (2018).

8. Gadjiev T., Aliev S., Galandarova Sh.: A priori estimates for solutions to Dirichlet boundary value problems for polyharmonic equations in generalized Morrey spaces, TWMS J. Pure Appl. Math. 9 (2), 231-242 (2018).

9. Grafakos L., Torres R.H.: Multilinear Calderón-Zygmund theory, Adv. Math. 165 (1), 124-164 (2002).

10. Grafakos L., Torres R.H.: On multilinear singular integrals of Calderón-Zygmund type, Publ. Mat. Vol. Extra, 57-91 (2002). 
11. Guliyev V.S.: Integral operators on function spaces on the homogeneous groups and on domains in $\mathbb{R}^{n}$, (Russian) Doctor's degree dissertation, Mat. Inst. Steklov, Moscow, 329 pp. (1994).

12. Guliyev V.S.: Function spaces, Integral Operators and Two Weighted Inequalities on Homogeneous Groups. Some Applications, (Russian) Casioglu, Baku, 332 pp. (1999).

13. Guliyev V. S.: Boundedness of the maximal, potential and singular operators in the generalized Morrey spaces, J. Inequal. Appl. Art. ID 503948, 20 pp. (2009).

14. Guliyev V.S.: Generalized weighted Morrey spaces and higher order commutators of sublinear operators, Eurasian Math. J. 3 (3), 33-61 (2012).

15. Guliyev V.S., Ismayilova A.F.: Multi-sublinear maximal operator and multilinear singular integral operators on generalized Morrey spaces, Proc. Inst. Math. Mech. Natl. Acad. Sci. Azerb. 40 (2), 65-77 (2014).

16. Guliyev V.S., Omarova M.N., Ragusa M.A., Scapellato A.: Commutators and generalized local Morrey spaces, J. Math. Anal. Appl. 457 (2), 1388-1402 (2018).

17. Guliyev V.S., Guliyev R.V., Omarova, M.N.: Riesz transforms associated with Schrödinger operator on vanishing generalized Morrey spaces, Appl. Comput. Math. 17 (1), 56-71 (2018).

18. Guliyev V.S., Akbulut A., Celik S., Omarova M.N.: Higher order Riesz transforms related to Schrdinger type operator on local generalized Morrey spaces, TWMS J. Pure Appl. Math. 10 (1), 58-75 (2019).

19. Ibrahimov E.J., Dadashova G.A., Ekincioglu S.E.: On the boundedness of the $G$ maximal operator and G-Riesz potential in the generalized G-Morrey spaces, Trans. Natl. Acad. Sci. Azerb. Ser. Phys.-Tech. Math. Sci. 40 (1), Mathematics, 111-125 (2020).

20. Lin Yan, Lu Shanzhen: Multlinear Calderón-Zygmund operator on Morrey type spaces, Anal. Theory Appl, 22, 387-400 (2006).

21. Mizuhara T.: Boundedness of some classical operators on generalized Morrey spaces, Harmonic Analysis (S. Igari, Editor), ICM 90 Satellite Proceedings, Springer - Verlag, Tokyo, 183-189 (1991).

22. Moen K.: Weighted inequalities for multilinear fractional integral operators, Collect. Math, 60, 213-238 (2009).

23. Morrey C.B.: On the solutions of quasi-linear elliptic partial differential equations, Trans. Amer. Math. Soc, 43, 126-166 (1938).

24. Nakai E.: Hardy-Littlewood maximal operator, singular integral operators and Riesz potentials on generalized Morrey spaces, Math. Nachr, 166, 95-103 (1994).

25. Rahimova K.R.: Characterizations of parabolic fractional integral operators on generalized parabolic Morrey spaces, Trans. Natl. Acad. Sci. Azerb. Ser. Phys.-Tech. Math. Sci. 36 (4), Mathematics, 156-166 (2016).

26. Sawano Y.: A thought on generalized Morrey spaces, J. Indonesian Math. Soc. 25 (3), 210-281 (2019). 